\title{
A THERMODYNAMIC MECHANISM BEHIND AN ACTION POTENTIAL AND BEHIND ANESTHESIA
}

\author{
BINYAM T. KASSAHUN \\ Department of Physics, East Carolina University \\ Greenville, NC 27858, USA \\ BTK1130@ecu.edu \\ ALEXANDER K. MURASHOV \\ Brody School of Medicine 6N-74, East Carolina University \\ 600 Moye Blvd., Greenville, NC 27834, USA \\ murashoval@ecu.edu \\ MARTIN BIER \\ Department of Physics, East Carolina University \\ Greenville, NC 27858, USA \\ bierm@ecu.edu \\ Received 26 August 2009 \\ Revised 8 April 2010
}

\begin{abstract}
It has been proposed that an action potential going through a nerve cell is not merely an electrochemical phenomenon, but also involves a traveling wave of compression and partial freezing of the lipid bilayer membrane. Below we present the results of experiments that are intended to discriminate between the electrochemical and the thermodynamic mechanism. We find that a nerve that is affected by an anesthetic can nevertheless reach the same compound action potential as an unaffected nerve when it receives a higher stimulus voltage. The result is hard to reconcile with the electrochemical HodgkinHuxley model and consistent with the thermodynamic mechanism.
\end{abstract}

Keywords: Action potential; anesthesia; cell membrane fluidity.

\section{Introduction}

The Hodgkin-Huxley $(\mathrm{HH})$ model describes how a signal propagates through a nerve. The model makes sense of a lot of phenomena. ${ }^{1}$ However, some puzzles remain. The purely electrochemical HH model gives, for instance, no good explanation for how anesthetics work. Since the early 1900s it has been known that a substance is a better anesthetic if it has a higher solubility in olive oil. This is called the Meyer-Overton Rule. ${ }^{2}$ The correlation between anesthetic potency and olive oil solubility is valid over several orders of magnitude. Many substances are anesthetics and oil solubility appears to be the only factor determining anesthetic potency. 
The HH model, furthermore, makes little sense of the anesthetic potency of xenon. Xenon gas (Xe) is chemically inert and it is hard to conceive of a way xenon would establish lasting bonds with sodium channels or other membrane proteins that are involved in the $\mathrm{HH}$ mechanism.

Recently, a model has been developed that can account for the Meyer-Overton rule and for a number of other unexplained observations on action potential propagation. $^{3,4}$ The bottom line message of these publications is that the $\mathrm{HH}$ model is, at best, incomplete. There is good evidence now, that the propagating "action potential" also involves a thermomechanical wave of partial "freezing."

A cell membrane is only two molecular layers thick and it is held together by hydrophobic and hydrophilic forces. Under physiological conditions the phospholipid molecules that constitute the membrane can freely diffuse in the plane of the membrane and, in this sense, the membrane is essentially a liquid. It has long been known that cells operate at a temperature that is only slightly above the temperature at which the cell membrane "freezes." When the membrane freezes, it basically turns into a gel: the lipid tails of the phospholipid molecules get entangled, molecules no longer diffuse freely, and the membrane is, for all practical purposes, a two-dimensional solid. Heimburg and Jackson propose in Ref. 3 that the action potential traveling along a nerve cell involves a compression wave; a compression wave that also involves a partial gellification. It is a mechanical and thermodynamic wave that can be compared somewhat to a wave that is going through a slinky. In Ref. 3 the process is modeled in terms of basic thermodynamics. The speed of the wave is derived from basic membrane parameters and indeed turns out to be very close to the speed of nerve propagation. With the Heimburg-Jackson (HJ) model it is easily explained how the Meyer-Overton rule ensues. The anesthetic that dissolves in the membrane lowers the freezing temperature of that membrane in the same way that salt lowers the freezing temperature of water. ${ }^{5}$ The decreased freezing temperature would mitigate the gellification wave that the action potential is in the HJ model.

Below we present the results of experiments that we conducted in order to corroborate whether predictions of the HJ model are borne out in biological reality. We excised bullfrog sciatic nerves and constructed a setup to trigger a signal on one end of the nerve and record the induced pulse on the other end of the nerve. The induced pulse is a sum over action potentials through many different nerve cells and it is called the compound action potential (CAP). We varied the stimulus voltage and we recorded the effect on CAP amplitude of two well-known anesthetics, xenon and procaine.

As was mentioned above, xenon is a chemically inert noble gas. It is hard to imagine how it would form an enduring bond with ion channels or other membrane proteins. The HJ model explains its anesthetic potency with the fact that, due to xenon being very nonpolar, a significant fraction dissolves in the membrane's lipid interior. Procaine, which is also known as novocaine, is assumed to block propagation of action potentials by blocking sodium channels on the intracellular 
side. ${ }^{1}$ It has good lipid solubility and this, presumably, helps it to cross the cell membrane.

\section{Materials and Methods}

We acquired bullfrogs (Rana catesbeiana) from Science Kit and Boreal Laboratories. These frogs measure between 10 and $15 \mathrm{~cm}$ in length and their sciatic nerves measure between 5 and $6 \mathrm{~cm}$ long. The frogs were housed at room temperature and they were fed live crickets.

Experiments were conducted according to animal protocol approved by IACUC at East Carolina University. After decapitation and pithing, the part of the nerve from the spinal cord to the ankle was excised and transferred to a petri dish containing a Ringer's solution. The nerve remained there for about 15 minutes before it was moved to the nerve chamber for the experiment.

The special nerve chamber for measurements was designed and built at the machine shop of the physics department of East Carolina University. The nerve is placed on the series of silver electrodes (see Fig. 1) and sprayed with Ringer's solution. At the bottom of the chamber, close to the nerve but not touching, a Ringer's solution keeps the nerve from drying out. During the experiment, the unit was covered. The stimulus was applied through two wires at the left. A Grass S48 unit was used for this purpose. On the right end of the chamber, the passing CAP was recorded. The output at the recording electrode was digitally stored and also viewed in real time on an oscilloscope.

Measurements started with a stimulus voltage of $400 \mathrm{mV}$. Ten stimuli were given at $0.2 \mathrm{~s}$ intervals and the response was recorded. Stimuli were biphasic and lasted $1 \mathrm{~ms}$. Subsequently, the stimulus voltage was increased by $50 \mathrm{mV}$. The experiment was ended once it was clear that the CAP had asymptotically reached its maximum value.

The effect of xenon was determined by putting a cover over the chamber and pulling a partial vacuum with a $200 \mathrm{cc}$ syringe. A tube was next attached to run

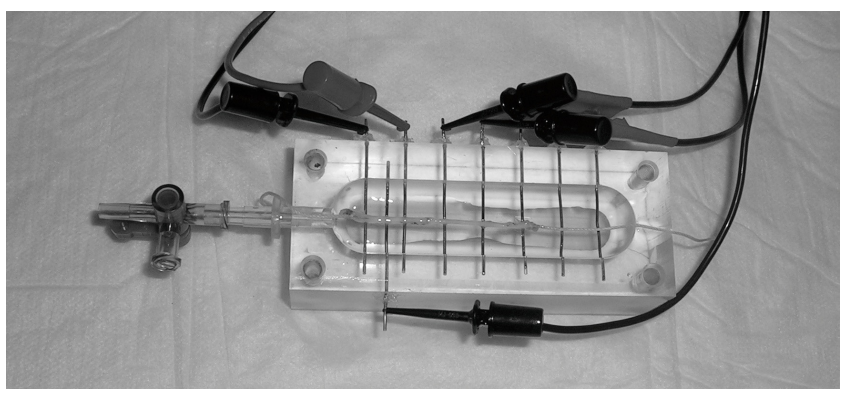

Fig. 1. An isolated sciatic nerve lying across the nerve chamber electrodes. The wires and electrodes on the left are used to give the stimulus. Further to the right, we record the compound action potential that is triggered by the stimulus. 
from a xenon cylinder to the chamber. A cylinder of $99.995 \%$ Xe was purchased from Matheson Trigas. Using the control valves, a constant flow of xenon gas was introduced into the chamber for $30 \mathrm{~s}$.

We also performed the experiment with a constant flow of argon instead of xenon. At atmospheric pressure, argon is not supposed to have any anesthetic effect. We ran this experiment in order to make sure that our method of "blowing" gas into the chamber was not drying out the nerve and responsible for the measured impairment. Indeed no effect was found with argon.

To study the effect of procaine, nerves were submerged in a petri dish containing $4 \times 10^{-4} \mathrm{M}$ of procaine before being moved to the chamber. A solution of $97 \%$ procaine hydrochloride was purchased from Sigma Aldrich. Six nerves were used to examine the effect of procaine and six nerves were used for control.

\section{Results and Discussion}

Figure 2 shows how, both without and with anesthetic, the CAP increases with the stimulus voltage. Working with the intact nerve, we could not control or even measure the concentration of anesthetic in the membrane or in the cell itself. However, Fig. 2 shows how our experiments, even for nerves from different frogs, lead to almost identical responses for the same stimulus. After removal of the xenon, the control behavior was reestablished within 5 minutes. After 2 hours in a Ringer's solution the procaine affected nerves also came back to their control behavior.

It is most remarkable that, in the presence of anesthetic, the same maximum CAP can actually be reached again. It apparently just requires a larger stimulus voltage. This observation is hard to reconcile with the $\mathrm{HH}$ mechanism. The action potential in the $\mathrm{HH}$ model is an all-or-none event. For the action potential to occur, it is only necessary that the stimulus voltage is above a threshold. Once the action potential is triggered, its amplitude only depends on the kinetic properties and densities of the involved ion channels and on the magnitude of the electrochemical potential between the intracellular fluid and the extracellular fluid. In the $\mathrm{HH}$ model, the transmembrane electrochemical potential fuels the autonomous dynamics of the action potential and the action potential is independent of the original magnitude of the stimulus. If a significant fraction of the channels is blocked, then the $\mathrm{HH}$ model predicts an action potential of compromised speed and amplitude. ${ }^{6}$ According to the $\mathrm{HH}$ model, the magnitude of the stimulus voltage should not matter in this. With the HH model, it is therefore hard to explain how a higher stimulus voltage "cures" the compromised ion channels and reestablishes the maximum CAP.

The observed full recovery of the CAP in an anesthetized nerve with a higher stimulus is consistent with the HJ model. Figure 3 shows how an electric stimulus brings the anesthetic-free membrane to below the freezing point and explains how, after the addition of anesthetic, the same trigger leads to only a partial freezing. An increase of the stimulus strength can again lead to complete freezing. 


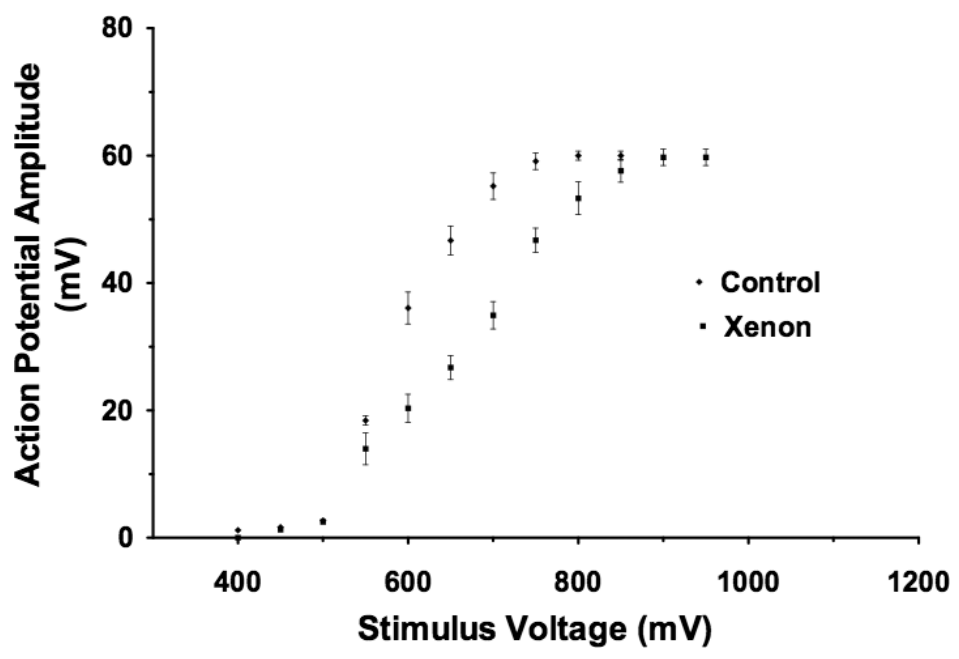

(a)

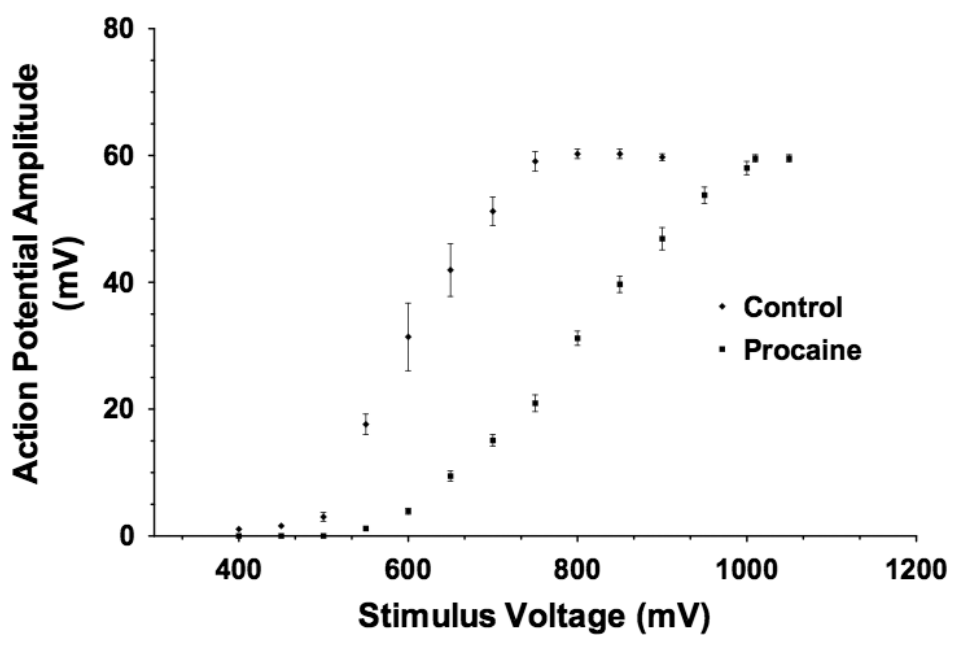

(b)

Fig. 2. (a) The amplitude of the compound action potential as a function of the stimulus voltage for a sciatic nerve with and without the anesthetic xenon. It is observed that both with and without the anesthetic the same amplitude is asymptotically approached for increasing stimulus voltage. But in the presence of anesthetic, a larger stimulus voltage is necessary to evoke the same response. (b) The same effect is measured with procaine as the anesthetic.

We, furthermore, see in Fig. 2 that xenon and procaine are similar in how they affect the CAP amplitude vs. stimulus behavior. This observation suggests that procaine's anesthetic potency may, to a larger extent than hitherto realized, rely on its membrane solubility and on the ensuing interference with the compressionand-freezing wave through the lowering of the freezing temperature. 


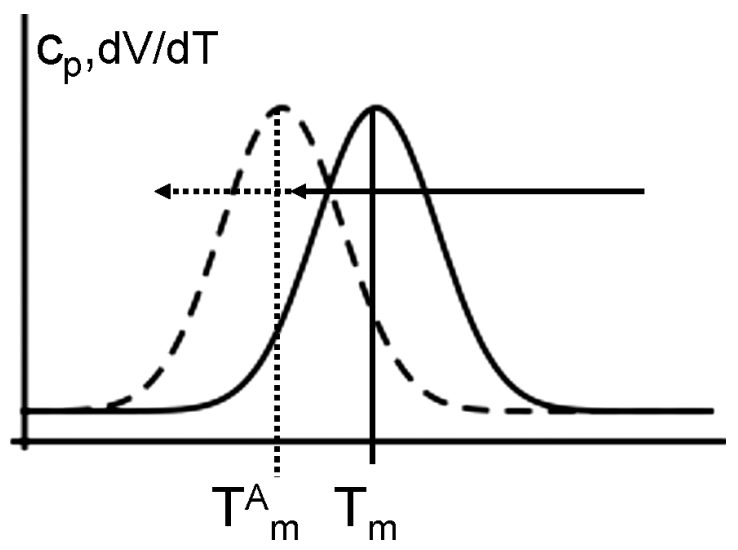

Fig. 3. Sketch of the heat capacity profile of a lipid bilayer membrane. The volume expansion coefficient, $d V / d T$, is generally proportional to the heat capacity $c_{p}$. The solid and dashed curve represent the membrane without and with anesthetic, respectively. $T_{m}$ is the maximum of the curve and represents the melting temperature. The electric stimulus that induces membrane freezing and triggers the thermoacoustic wave is represented with an arrow. A stimulus that is strong enough to induce complete freezing in the absence of anesthetic leads to partial freezing with anesthetic present. Increased stimulus (dashed arrow) will, in that case, again lead to complete freezing.

Though there is a good description of how a thermoacoustic freezing wave propagates through a lipid bilayer, ${ }^{3}$ there is no good quantitative model to relate the increased amplitude of the thermoacoustic freezing wave to the increased electric stimulus. Such a model would involve the piezoelectric properties of the cell membrane and these are only beginning to be researched. ${ }^{7}$

Some of the basic premises of the HJ model for the propagation of an action potential have been experimentally tested on artificial membranes. ${ }^{5}$ Our work constitutes the first test of the HJ model in a biological setting. As was mentioned before, our results are mostly qualitative in the sense that we could not control or measure the exact concentration of anesthetic in the membrane or the cell. A more rigorous study should also involve a measurement of the membrane's heat capacity profile with and without anesthetic.

\section{Acknowledgments}

We are grateful to Thomas Heimburg for useful discussions. We also thank Teresa Lever for invaluable help with the experiments.

\section{References}

1. B. Hille, Ionic Channels of Excitable Membranes (Sinauer, 1992).

2. W. J. Moore, Physical Chemistry, 5th edn. (Prentice Hall Inc., 1972).

3. T. Heimburg and A. D. Jackson, Proc. Natl. Acad. Sci. USA 102, 9790 (2005). 
4. T. Heimburg and A. D. Jackson, Biophys. J. 92, 3159 (2007).

5. T. Heimburg, Thermal Biophysics of Membranes (Wiley-VCH Verlag, 2007).

6. J. G. Nichols, A. R. Martin and B. G. Wallace, From Neuron to Brain, 3rd edn. (Sinauer, 1992).

7. A. Jákli, J. Harden, C. Notz and C. Bailey, Liquid Crystals 35(4), 395 (2008). 